Background and aims Systemic lupus erythematosus (SLE) is an autoimmune and inflammatory disease with multiple clinical manifestations including arthropathy. The severity of the articular involvement or deformity to an erosive deforming arthropathy with severe functional disability. In rare cases a severe, erosive and deforming arthropathy, clinically indistinguishable from rheumatoid arthritis (RA) can be observed; this clinical entity is traditionally known as "Rhupus". It remains controversial whether Rhupus is a distinct entity, an overlap between SLE and RA or a serious articular involvement of SLE.

Methods Observational.

Results A 23 years old female presented with polyarthritis, vasculitis, and anaemia. She was having symmetrical inflammatory polyarthritis of both upper and lower limb for last 2 years and was having skin rash for last 1 year. She also gave history of recurrent oral ulcers, photosensitivity and alopecia. On Investigation, revealed anaemia, alopecia, oral ulcer, vasculitis and active synovitis of both elbows, wrists, hands, knees and ankle joints. Her report showed raised of inflammatory parameter, normochomic normocytic anaemia. Further work up showed positive result in RF and anti-IgG. Also showed positive results on some antigens ANA profile. She was started on prednisolone, Metotrexate, folic acid, hydroxychloroquine, calcium, vitamin D and Natrium Diclofenac.

Conclusions Rhupus Syndrome is a rare syndrome. Currently, Rhupus remains an entity not perfectly known, but the pathogenesis, the autoantibody positivity, the radiological manifestations and therapy all support the idea that it is really an overlap syndrome between SLE and RA, although its pathogenesis still remains to be fully understood .

\section{ACTIVE ARTHRITIS IS ASSOCIATED WITH 14-3-BETA TITRE IN PATIENTS WITH SYSTEMIC LUPUS ERYTHEMATOSUS}

C Hitchon*, D Robinson, H El-Gabalawy, A Tisseverasinghe, A Man, C Peschken. University of Manitoba, Internal Medicine, Winnipeg, Canada

\subsection{6/lupus-2017-000215.215}

Background and aims Non-erosive arthritis is common in systemic lupus erythematosus (SLE). 14-3-3 eta, a chaperone protein that activates pro-inflammatory pathways is emerging as a novel biomarker for erosive Rheumatoid Arthritis. We investigated clinical associations of serum 14-3-3 eta in SLE focusing on arthritis.

Methods Sociodemographics, ACR criteria, and SLEDAI were recorded. Arthritis, assessed by the SLEDAI, was categorised as active $(n=78)$, inactive $(n=138)$ and never present $(n=49)$. Serum 14-3-3 eta was measured by ELISA; titres above $0.19 \mathrm{ng} / \mathrm{ml}$ were considered positive. We report descriptive statistics and logistic regression models testing the association of 14-3-3eta with arthritis state.

Results SLE patients $(n=265)$ were mainly female (92\%), Caucasian (67\%) with a mean (SD) age of 51.7 (14) years, and median $(25 \%, 75 \%)$ disease duration of $8(4,10)$ years, number ACR criteria of $6(5,7)$, and SLEDAI of 4 (2,7). 241 (81\%) had active or inactive arthritis. 14-3-3 eta positivity was similar across the three arthritis groups (active 22/78 (28\%), inactive $27 / 138$ (20\%), never present $10 / 49$ (20\%) with a median $(25 \% 75 \%)$ titre of $0.6 \mathrm{ng} / \mathrm{ml}(0.34,1.82)$. The highest quartile of $14-3-3$ eta associated with active arthritis (OR 3.6 $(95 \%$ CI $1.33,9.98) \mathrm{p}-0.012)$ after adjusting for ethnicity and SLEDAI. There were no differences in 14-3-3 eta positivity for other lupus criteria nor correlation of 14-3-3 eta titer with number of ACR criteria or SLEDAI.

Conclusions 14-3-3 eta titers are highest in lupus patients with active arthritis suggesting a higher risk for more severe arthritis. Further work will explore the associations of $14-3-3$ eta in lupus with erosive arthritis.

\section{ACUTE PANCREATITIS AS AN INITIAL MANIFESTATION OF SYSTEMIC LUPUS ERYTHEMATOSUS : TWO CASE REPORTS}

CG Khor*, M Tan, PS Ong. hospital raja permaisuri bainun, Rheumatology Unit- Internal Medicine Department, ipoh, Malaysia

\subsection{6/lupus-2017-000215.216}

Background and aims Acute pancreatitis is a rare initial presentation of systemic lupus erythematosus.

Methods We report two cases of Malay patients who presented with fever, abdominal pain, ascites and high level of pancreatic enzymes. They were diagnosed with acute pancreatitis associated with systemic lupus erythematosus.

Results First patient is a Malay male patient presented to us late. He had severe acute pancreatitis with severe hyperbilirubinemia, lupus nephritis and autoimmune haemolytic anaemia. He died despite maximal therapy was provided. The second case is a Malay lady who came to us early responded well to high dose corticosteroids and discharged well.

Conclusions The mortality rate of lupus-associated pancreatitis is higher than in non-lupus associated pancreatitis. Early awareness of lupus-associated pancreatitis as an initial manifestation of systemic lupus erythematosus is crucial in managing and improving outcome in lupus patients.

\section{PRIOR SUN EXPOSURE AND SKIN-SPECIFIC AUTO- ANTIBODIES ARE ASSOCIATED WITH SKIN DISEASE IN SYSTEMIC LUPUS ERYTHEMATOSUS}

${ }^{1} \mathrm{~T}$ Khosravi-Hafshejani* ${ }^{2} \mathrm{M}$ Ghoreishi, ${ }^{3} \mathrm{~A}$ Kariminia, ${ }^{2} \mathrm{~S}$ Kalia, ${ }^{4} \mathrm{~A}$ Avina-Zubieta, 4J Reynolds, ${ }^{2} \mathrm{JP}$ Dutz. 'University of British Columbia, Undergraduate Medical ProgramFaculty of Medicine, Vancouver, Canada; ' University of British Columbia, Dermatology and Skin Science, Vancouver, Canada; ${ }^{3}$ University of British Columbia, Pediatrics- Division of Haematology and Oncology, Vancouver, Canada; ${ }^{4}$ University of British Columbia, MedicineDivision of Rheumatology, Vancouver, Canada

\subsection{6/lupus-2017-000215.217}

Background and aims Almost $80 \%$ of systemic lupus erythematosus (SLE) patients manifest lupus-specific skin lesions. A pathogenic link between skin inflammation and SLE has been proposed. We hypothesised that skin-directed antibodies are present in SLE and are associated with a history of significant sun-exposure.

Methods Blood was collected from three patient populations; SLE with a history of lupus-specific skin lesions as cases $(n=15)$, SLE without a history of skin lesions $(n=7)$ and atopic dermatitis $(n=6)$ as controls. Serum antid-esmoglein-3 antibodies were measured by ELISA. Peripheral blood 